\title{
Airy kernel with two sets of parameters in directed percolation and random matrix theory.
}

\author{
Alexei Borodin * and Sandrine Péché ${ }^{\dagger}$
}

January 19, 2014

\begin{abstract}
We introduce a generalization of the extended Airy kernel with two sets of real parameters. We show that this kernel arises in the edge scaling limit of correlation kernels of determinantal processes related to a directed percolation model and to an ensemble of random matrices.
\end{abstract}

\section{Introduction and results}

The Airy kernel is one of the most fundamental objects of Random Matrix Theory. The determinantal random point process governed by the Airy kernel describes the behavior of the largest eigenvalues of large Gaussian Hermitian random matrices (a.k.a. GUE - Gaussian Unitary Ensemble), see [6], 10], 25], last passage time in directed percolation models in a quadrant [17, asymptotics of the longest increasing subsequences of random permutations [1, and it also appears in many other problems whose list is too long to be included here.

The Airy kernel has a time-dependent version usually referred to as the extended Airy kernel. Originally obtained in 24] via asymptotics of a polynuclear growth model in $1+1$ dimensions, the extended Airy kernel arises in virtually every problem where the usual Airy kernel comes up, provided that the probability measure in question is equipped with a natural

\footnotetext{
*Mathematics 253-37, Caltech, Pasadena, CA 91125, USA; Email:borodin@caltech.edu and Institute for Information Transmission Problems, Moscow, Russia

${ }^{\dagger}$ Institut Fourier, 100 Rue des maths, 38402 Saint Martin d'Heres, France; E-mail: Sandrine.Peche@ujf-grenoble.fr Temporary address while this work was done: Department of Mathematics, University of California at Davis, One Shields Ave., Davis, CA 95616, USA.
} 
Markov dynamics that preserves the measure. In particular, it describes the edge scaling limit of Dyson's Brownian motion on GUE, the change in the quadrant last passage time when the observation point moves [18], and edge behavior of large random partitions under the Plancherel dynamics related to the longest increasing subsequences of random permutations [4]. The Extended Airy kernel has also appeared in a much earlier note [19], and we are very grateful to a referee for pointing this out.

In [2] it was demonstrated that the Airy kernel is not stable in the sense that it can be naturally viewed as a point in a family of Airy-like kernels indexed by a finite set of real numbers. In the context of GUE, those are the (scaled) eigenvalues of a deterministic perturbation of finite rank, and in the percolation context the parameters correspond to a few defective rows or columns in the quadrant. For Wishart ensembles of random matrices, the same family of kernels was later obtained in [7. Time-dependent extensions of kernels from this family appeared in the recent work [14] on asymptotics of the totally asymmetric simple exclusion process (TASEP).

The main goal of this note is to introduce a new Airy-like time-dependent correlation kernel with two sets of real parameters. We obtain it as a limit of a directed percolation in a quadrant which has both defective rows and columns. We also show that the "static" version of the kernel arises in the edge scaling limit of a certain Wishart-like ensemble of random matrices. We were unable to obtain the extended version via random matrices but we do believe that it should be possible. Our kernel generalizes all the kernels mentioned above.

Let us describe our results in more detail.

Consider a directed percolation model with exponential waiting times defined as follows. Let $\pi_{1}, \ldots, \pi_{p}, \hat{\pi}_{1}, \ldots, \hat{\pi}_{p}$ be fixed real numbers such that $\pi_{i}+\hat{\pi}_{j}>0$ for any $1 \leq i, j \leq p$. Let $W=\left(W_{i j}\right)_{i, j=1, \ldots, p}$ be a $p \times p$ array of independent exponential random variables with $\mathbb{E}\left(W_{i j}\right)=\left(\pi_{i}+\hat{\pi}_{j}\right)^{-1}$. For any $1 \leq N \leq p$, we consider the so-called last passage time in this percolation model:

$$
Y(N, p):=\max _{P \in \Pi} \sum_{(i j) \in P} W_{i j},
$$

where $\Pi$ is the set of up-right paths from $(1,1)$ to $(N, p)$. The random variable $Y(N, p)$ has a natural interpretation in terms of queuing theory, due to the result of [13]. This is the exit time of the $p$ th customer in a series of $N$ files where the service times $W_{i j}$ depend on both the file and the customer. This random variable also has an interpretation in terms of TASEP, which 
is a model of interacting particles on $\mathbb{Z}$. One starts with the initial configuration $\eta_{0}(i)=1_{\mathbb{Z}_{-}}(i)$, meaning that only the negative sites are occupied. Then, if the site $i+1$ is unoccupied, the particle at site $i$ jumps to site $i+1$ after a random waiting time. The waiting times are independent exponential random variables whose parameters depend on the particle and the number of jumps already performed by this particle. One can think of $W_{i j}$ as of the waiting time of $i$ th particle from the right performing the jump number $j$.

We first prove the following result.

Let $X_{N}$ be a $p \times N$ random matrix with independent complex Gaussian entries

$$
X_{i j} \sim \mathcal{N}\left(0, \frac{1}{\pi_{i}+\hat{\pi}_{j}}\right) .
$$

Theorem 1. Let $\lambda_{1}$ be the largest eigenvalue of $X_{N} X_{N}^{*}$. Then, for any $x$,

$$
\mathbb{P}(Y(N, p) \leq x)=\mathbb{P}\left(\lambda_{1} \leq x\right) .
$$

A natural question is then to investigate the above connection as a process. Consider a sequence of growing random matrices $\left(X_{k}\right)_{k=1, \ldots, p}$ where $X_{k+1}$ is obtained from $X_{k}$ by adding one column with random Gaussian entries (with the appropriate variance). We can then consider the joint distribution of the largest eigenvalues of the random matrices $X_{k} X_{k}^{*}, 1 \leq k \leq p$. Simultaneously, one can consider the joint distribution of the random variables $Y(k, p), 1 \leq k \leq p$. Are these joint distributions the same? We cannot establish that the equality actually holds, due to the fact that the computation of the joint eigenvalue distribution of the random matrices $X_{k} X_{k}^{*}$ is not an easy task 1 Nevertheless, we can study a determinantal point process which occurs naturally in both models and obtain a new limiting correlation kernel, which generalizes the extended Airy kernel.

Let $J_{1}, J_{2}$ be given integers, and $X=\left\{x_{1}, x_{2}, \ldots, x_{J_{1}}\right\}, Y=\left\{y_{1}, y_{2}, \ldots, y_{J_{2}}\right\}$ be given sets of real numbers satisfying $x_{i}>y_{j}$ for any $1 \leq i \leq J_{1}$ and any $1 \leq j \leq J_{2}$. Let $\gamma$ and $\Gamma$ be the contours defined on Figure 1 below.

Denote by $K_{A i}\left(t_{1}, x ; t_{2}, y\right)$ the extended Airy kernel $K_{A i}\left(t_{1}, x ; t_{2}, y\right)=\left\{\begin{array}{l}\int_{0}^{\infty} e^{-\lambda\left(t_{1}-t_{2}\right)} A i(y+\lambda) A i(x+\lambda) d \lambda, \text { if } t_{1} \geq t_{2}, \\ -\int_{-\infty}^{0} e^{-\lambda\left(t_{1}-t_{2}\right)} A i(y+\lambda) A i(x+\lambda) d \lambda, \text { if } t_{1}<t_{2} .\end{array}\right.$

\footnotetext{
${ }^{1}$ As was pointed out to us by Peter Forrester, the equality can be established in the degenerate case when all $\pi_{i}$ tend to the same constant using the techniques of [12, see Appendix to [1].
} 


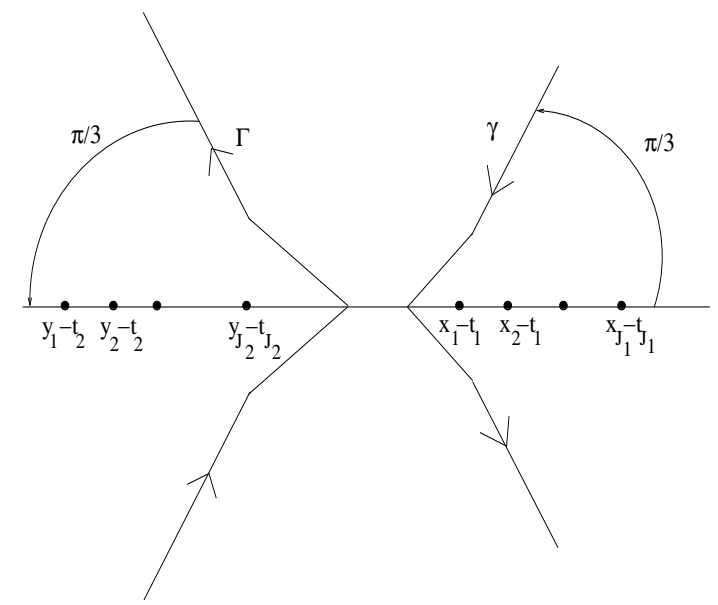

Figure 1: The contours of the parameterized extended Airy kernel.

Definition 1. The extended Airy kernel with two sets of parameters is defined by

$$
\begin{aligned}
& K_{A i ; X, Y}\left(t_{1}, x ; t_{2}, y\right)=K_{A i}\left(t_{1}, x ; t_{2}, y\right)+\frac{1}{(2 \pi i)^{2}} \int_{\gamma} d \sigma \int_{\Gamma} d \tau \\
& \frac{e^{y \tau-\tau^{3} / 3-x \sigma+\sigma^{3} / 3}}{\tau+t_{2}-\sigma-t_{1}}\left(\prod_{i=1}^{J_{1}} \frac{t_{2}+\tau-x_{i}}{t_{1}+\sigma-x_{i}} \prod_{i=1}^{J_{2}} \frac{t_{1}+\sigma-y_{i}}{t_{2}+\tau-y_{i}}-1\right),
\end{aligned}
$$

where the two contours $\gamma$ and $\Gamma$ are chosen as on Figure 1 .

Remark 1. For any fixed values of $t_{1}$ and $t_{2}$, the kernel of Definition 1 is a finite rank perturbation of the extended Airy kernel. Indeed, by a straightforward computation, one finds that

$$
\begin{gathered}
K_{A i ; X, Y}\left(t_{1}, x ; t_{2}, y\right)-K_{A i}\left(t_{1}, x ; t_{2}, y\right) \\
=\frac{1}{(2 i \pi)^{2}} \sum_{k_{1}+k_{2}=1}^{J_{1}+J_{2}} \int_{\gamma} d \sigma \int_{\Gamma} d \tau e^{y \tau-\tau^{3} / 3-x \sigma+\sigma^{3} / 3} \\
\sum_{1 \leq i_{1}<\cdots<i_{k_{1}} \leq J_{1}} \sum_{1 \leq j_{1}<\cdots<j_{k_{2}} \leq J_{2}} \frac{(-1)^{k_{2}}\left(\tau+t_{2}-\sigma-t_{1}\right)^{k_{1}+k_{2}-1}}{\prod_{l=1}^{k_{1}}\left(\sigma+t_{1}-x_{i_{l}}\right) \prod_{l^{\prime}=1}^{k_{2}}\left(\tau+t_{2}-y_{j_{l^{\prime}}}\right)} .
\end{gathered}
$$

Remark 2. One does not have to stop at considering finitely many perturbation parameters. By taking limits with number of $x_{i}$ 's and $y_{j}$ 's going to 
infinity, one arrives at the following kernel. Let $\left\{a_{i}^{ \pm}\right\}_{i=1}^{\infty}$ and $\left\{b_{i}^{ \pm}\right\}_{i=1}^{\infty}$ be four sequences of nonnegative numbers such that $\sum_{i=1}^{\infty}\left(a_{i}^{ \pm}+b_{i}^{ \pm}\right)<\infty$, and let $c^{ \pm}$be two positive numbers. Set

$$
\Phi_{a, b, c}(z)=e^{c^{+} z+c^{-} z^{-1}} \prod_{i=1}^{\infty} \frac{\left(1+b_{i}^{+} z\right)\left(1+b_{i}^{-} z^{-1}\right)}{\left(1-a_{i}^{+} z\right)\left(1-a_{i}^{-} z^{-1}\right)} .
$$

Then the kernel

$$
\begin{aligned}
& K_{A i ; a, b, c}\left(t_{1}, x ; t_{2}, y\right)=K_{A i}\left(t_{1}, x ; t_{2}, y\right) \\
& \quad+\frac{1}{(2 \pi i)^{2}} \int_{\gamma} d \sigma \int_{\Gamma} d \tau \frac{e^{y \tau-\tau^{3} / 3-x \sigma+\sigma^{3} / 3}}{\tau+t_{2}-\sigma-t_{1}}\left(\frac{\Phi_{a, b, c}\left(\sigma+t_{1}\right)}{\Phi_{a, b, c}\left(\tau+t_{2}\right)}-1\right),
\end{aligned}
$$

where the contours are chosen so that all points $1 / a_{i}^{+}-t_{1}$ and $a_{i}^{-}-t_{1}$ are to the right of $\gamma$, and all points $-1 / b_{i}^{+}-t_{2}$ and $-b_{i}^{-}-t_{2}$ are to the left of $\Gamma$, is readily seen to be a limit of kernels of Definition 1. Interestingly enough, functions (6) also parameterize stationary extensions of the discrete sine kernel, see [3]. They also appear as generating functions of totally positive doubly infinite sequences [8], and as indecomposable characters of the infinite-dimensional unitary group, see [22] and references therein.

The main result of this note is the proof that the kernel $K_{A i ; X, Y}$ arises as a scaling limit of correlation kernels of the determinantal point processes related to the directed percolation model in a quadrant, defined as follows. Let $0<t<1$ be a given real number and assume that there exist two integers $J_{1}, J_{2}$, real numbers $x_{i}, 1 \leq i \leq J_{1}, y_{j}, 1 \leq j \leq J_{2}$ independent of $p$ such that:

$$
\begin{gathered}
\pi_{i}=\frac{\sqrt{t}}{\sqrt{t}+1}+\frac{x_{i}}{\alpha p^{1 / 3}}, \quad i=1, \ldots, J_{1} ; \quad \pi_{i}=1, i>J_{1} ; \\
\hat{\pi}_{j}=-\frac{\sqrt{t}}{\sqrt{t}+1}-\frac{y_{j}}{\alpha p^{1 / 3}}, \quad j=1, \ldots, J_{2}, \quad \hat{\pi}_{j}=0, j>J_{2} .
\end{gathered}
$$

We consider the associated directed percolation model (1) and prove the following result.

Set

$$
\alpha_{s}=t+2 \frac{(t(1+\sqrt{t}))^{2 / 3}}{p^{1 / 3}} s, Y_{s}=Y\left(p \alpha_{s}, p\right) \text { and } \sigma_{s}=p\left(1+\sqrt{\alpha_{s}}\right)^{2} .
$$

Let also $\mathcal{A}_{s}^{\text {ext }}$ be the stochastic process whose finite dimensional distributions are given by

$$
\mathbb{P}\left(A_{t_{1}}^{e x t} \leq \xi_{1}, \ldots, A_{t_{m}}^{e x t} \leq \xi_{m}\right)=\operatorname{det}\left(I-f K_{A i ; X, Y} f\right)_{L^{2}\left(\Gamma^{m} \times \mathbb{R}^{m}\right)},
$$


where $f\left(t_{j}, x\right)=1_{x \geq \xi_{j}}$ and $\Gamma^{m}=\left\{t_{1}, \ldots, t_{m}\right\}$. Here $\Gamma^{m} \times \mathbb{R}^{m}$ is equipped with the product measure $d \mu \otimes d \lambda$ where $d \mu$ is the counting measure and $d \lambda$ denotes the Lebesgue measure.

Theorem 2. As $p \rightarrow \infty$,

$$
p^{-1 / 3} t^{1 / 6}(1+\sqrt{t})^{4 / 3}\left(Y_{s}-\sigma_{s}\right) \rightarrow \mathcal{A}_{s}^{e x t},
$$

in the sense of convergence of finite dimensional distributions.

The paper is organized as follows. In Sections 2 and 3, we study the directed percolation model and its connection to the random matrix model defined in (2). This connection is established using the Robinson-SchenstedKnuth correspondence. In Section 4, we study the correlation function of the point process induced by the joint distribution of $\{Y(k, p)\}_{1 \leq k \leq p}$. Last, in Section 5, we consider asymptotics of these correlation functions thus obtaining the kernel of Definition 1 .

Acknowledgments. The first named author was partially supported by the NSF grants DMS-0402047 and DMS-0707163. The second named author was visiting UC Davis when this paper was written. She would like to thank especially A. Soshnikov who allowed her to spend a great year at UC Davis and encouraged this work.

The authors are also very grateful to Peter Forrester for a number of valuable remarks.

\section{Last passage time in percolation models with exponential waiting times.}

We start with some reminders. We denote a partition by $\lambda$ throughout the paper. A partition is an infinite sequence of non-negative integers $\left(\lambda_{1}, \lambda_{2}, \ldots, \lambda_{N}, \ldots\right)$, where $\lambda_{1} \geq \lambda_{2} \geq \cdots \geq \lambda_{N} \geq \cdots$, with finitely many nonzero entries. The Schur measure introduced in [21] is a measure on partitions which assigns to a given partition $\lambda$ a weight as follows. Let $a_{1}, \ldots, a_{N}$ and $b_{1}, \ldots, b_{p}$ be given nonnegative real numbers. In what follows we assume that $a_{i} b_{j}<1$ for any $i, j$.

Definition 2. The Schur measure with parameters $a=\left(a_{1}, \ldots, a_{N}\right)$ and $b=\left(b_{1}, \ldots, b_{p}\right)$ is a probability measure $\mathcal{M}$ on partitions assigning to a partition $\lambda$ the weight

$$
\mathcal{M}(\lambda)=\frac{1}{Z} s_{\lambda}(a) s_{\lambda}(b)
$$


Here $Z$ is a constant, and $s_{\lambda}$ denotes the Schur symmetric functions parameterized by $\lambda$.

The normalizing constant $Z$ is computed by the well-known Cauchy identity for Schur functions, which implies $Z=\prod_{1 \leq i \leq p, 1 \leq j \leq N}\left(1-a_{i} b_{j}\right)$.

The Schur measure naturally occurs in some directed percolation models, as we now recall. Let $W=\left(w_{i j}\right)_{1 \leq i \leq p, 1 \leq j \leq N}$ be a $p \times N$ random matrix with independent entries with the geometric distribution:

$$
\mathbb{P}\left(w_{i j}=n\right)=\left(1-a_{i} b_{j}\right)\left(a_{i} b_{j}\right)^{n}, \text { for any } n=0,1,2, \ldots .
$$

One can associate to this random matrix the so-called last passage time in a directed percolation model defined by

$$
L(N, p):=\max _{P \in \Pi} \sum_{(i j) \in P} w_{i j}
$$

where $\Pi$ is the set of up-right paths from $(0,0)$ to $(p, N)$. This last passage time can also be understood as the exit time of the $N$ th customer in a series of $p$ files with independent geometric waiting times of expectation depending on both the files and customer.

The distribution of the random variable (8) can be conveniently expressed in terms of the Schur measure, as observed by K. Johansson in [17, see also [18. He showed that

$$
\mathbb{P}(L(N, p) \leq n)=\frac{1}{Z} \sum_{\lambda_{1} \leq n} s_{\lambda}(a) s_{\lambda}(b) .
$$

This is a corollary of a more general fact that the Schur measure with parameters $a$ and $b$ is the image of the random integer valued matrix $W$ under the Robinson-Schensted-Knuth correspondence, see [17, [18].

We now turn to the description of a continuous version of the Schur measure. Another treatment of the same object can be found in Appendix A to [12. Set

$$
a_{i}=1-\frac{\pi_{i}}{L}, i=1, \ldots, p, \text { and } b_{j}=1-\frac{\hat{\pi}_{j}}{L}, j=1, \ldots, N .
$$

It is quite clear that the distribution random variable $Y_{L}(N, p):=\frac{1}{L} L(N, p)$ should converge as $L \rightarrow \infty$ to that of the last passage time in a percolation 
model with i.i.d. exponential random variables with expectations $1 /\left(\pi_{i}+\hat{\pi}_{j}\right)$. Define $Y(N, p)$ as in (11). Taking the limit $L \rightarrow \infty$ in (9) and denoting $x_{i}=\lambda_{i} / L$, one readily obtains in the case where $N=p$ that

$$
\mathbb{P}(Y(p, p) \leq x)=\frac{1}{Z_{p, p}} \int_{I^{p}} \operatorname{det}\left(e^{-\pi_{i} x_{j}}\right)_{i, j=1}^{p} \operatorname{det}\left(e^{-\hat{\pi}_{i} x_{j}}\right)_{i, j=1}^{p} \prod_{i=1}^{p} d x_{i},
$$

where $Z_{p, p}=\operatorname{det}\left(\frac{1}{\pi_{i}+\hat{\pi}_{j}}\right)_{i, j=1}^{p}$ and $I=[0, x]$. The probability measure defined by the density

$$
\frac{1}{Z_{p, p}} \operatorname{det}\left(e^{-\pi_{i} x_{j}}\right)_{i, j=1}^{p} \operatorname{det}\left(e^{-\hat{\pi}_{i} x_{j}}\right)_{i, j=1}^{p}
$$

is the continuous version of the Schur measure.

The case $N<p$ can be handled via the limit transition $b_{j} \rightarrow 0, j=$ $N+1, \ldots, p$, from the case $N=p$. The analog of (10) reads

$$
\mathbb{P}(Y(N, p) \leq x)=\frac{1}{Z_{N, p}} \int_{I^{N}} \operatorname{det}\left(f_{i}\left(x_{j}\right)\right)_{i, j=1}^{N} \operatorname{det}\left(e^{-\hat{\pi}_{i} x_{j}}\right)_{i, j=1}^{N} \prod_{i=1}^{p} d x_{i},
$$

with

$$
f_{k}(x)=\frac{1}{2 \pi i} \oint \frac{u^{k-1} e^{-u x} d u}{\prod_{j=1}^{p}\left(u-\pi_{j}\right)}, \quad k=1, \ldots, N,
$$

and the integration contour going around the poles $\pi_{1}, \ldots, \pi_{p}$.

The expression $\operatorname{det}\left(f_{i}\left(x_{j}\right)\right)_{i, j=1}^{N}$ can be obtained as the limit of the JacobiTrudi formula for $s_{\lambda}(a)$; it is also the limit, up to a constant, of the ratio

$$
\frac{\operatorname{det}\left(e^{-\pi_{i} x_{j}}\right)_{i, j=1}^{p}}{\prod_{N+1 \leq i<j \leq p}\left(x_{i}-x_{j}\right)}
$$

as $x_{N+1}, \ldots, x_{p}$ converge to 0 .

\section{A random matrix model associated to the con- tinuous version of the Schur measure.}

The goal of this section is to prove Theorem 1

Let $X_{N}$ be a $p \times N$ random matrix as in (2). Set then $M_{N}=X_{N} X_{N}^{*}$. Thus defined random matrix ensemble is a natural generalization of the much studied complex Wishart ensemble. In what follows we call it the generalized Wishart ensemble. We show that the probability distribution of the largest eigenvalue of $M_{N}$ has a density given by the right-hand side of (11). 
Let us first consider the case where $p=N$. Similarly to the case of the ordinary Wishart ensemble, one sees that the generalized Wishart ensemble is defined by the probability density with respect to Lebesgue measure $L_{p}$ on the space of complex matrices of size $p \times p$ :

$$
\frac{d \mathbb{P}\left(X_{p}\right)}{d L_{p}}=\text { const }_{p} \exp \left\{-\operatorname{Tr} S_{1} X_{p} X_{p}^{*}-\operatorname{Tr} S_{2} X_{p}^{*} X_{p}\right\},
$$

where $S_{1}=\operatorname{diag}\left(\pi_{1}, \ldots, \pi_{p}\right), S_{2}=\operatorname{diag}\left(\hat{\pi}_{1}, \ldots, \hat{\pi}_{p}\right)$, and const $_{p}$ is a positive constant. Just as for complex Wishart ensembles with non identity covariance matrix, the joint eigenvalue distribution of the generalized Wishart ensembles can be explicitly computed.

Denote by $x_{1} \geq x_{2} \geq \cdots \geq x_{p}$ the ordered eigenvalues of the sample covariance matrix $X_{p} X_{p}^{*}$. Note that these eigenvalues are also the squared singular values of $X_{p}$. Let $f\left(x_{1}, \ldots, x_{p}\right)$ denote the density with respect to Lebesgue measure of the joint eigenvalue distribution induced by the generalized Wishart ensemble.

Proposition 1. One has

$$
f\left(x_{1}, \ldots, x_{p}\right)=\frac{1}{Z_{p, p}} \operatorname{det}\left(e^{-\pi_{i} x_{j}}\right)_{i, j=1}^{p} \operatorname{det}\left(e^{-\hat{\pi}_{j} x_{k}}\right)_{j, k=1}^{p} .
$$

Proof of Proposition 1. Introduce the polar decomposition of the $p \times p$ matrix $X_{p}$ : One has

$$
X=U D V \text { with } U \in \mathbb{U}(p), \quad D=\operatorname{diag}\left(\sqrt{x_{1}}, \ldots, \sqrt{x_{p}}\right), \text { and } V \in \mathbb{U}(p) .
$$

The joint eigenvalue distribution induced by the probability measure (13) can now be computed thanks to the celebrated Itzykson-Zuber-HarischChandra (IZHC) integral. We have

$$
f\left(x_{1}, \ldots, x_{p}\right)=\text { const } \cdot V(x)^{2} \int_{\mathbb{U}(p)} e^{-\operatorname{Tr} S_{1} U D^{2} U^{*}} d U \int_{\mathbb{U}(p)} e^{-\operatorname{Tr} S_{2} V D^{2} V^{*}} d V .
$$

In the above expression, $V(x)$ is the Vandermonde determinant: $V(x)=$ $\prod_{i<j}\left(x_{i}-x_{j}\right)$. The IZHC formula yields

$$
\int_{\mathbb{U}(p)} e^{-\operatorname{Tr}_{1} U D^{2} U^{*}} d U=\frac{\operatorname{det}\left(e^{-\pi_{i} x_{j}}\right)_{i, j=1}^{p}}{V(x) V(\pi)} .
$$

The proof that the largest eigenvalue of $X_{N} X_{N}^{*}$ has the same distribution as the random variable $Y(N, p)$ can now be obtained from Proposition 1 and formulas of the previous section by the limit transition $\hat{\pi}_{N+1}, \ldots, \hat{\pi}_{p} \rightarrow \infty$.

This finishes the proof of Theorem 1 . 


\section{A continuous version of the Schur Process}

In this section, we define a random point process which is a continuous version of the Schur process introduced in 23. We consider the probability distribution on $\prod_{i=1}^{p} \mathbb{R}_{+}^{i}$ with density w.r.t. Lebesgue measure given by

$$
\frac{1}{Z_{p}} \operatorname{det}\left(e^{-\pi_{i} x_{j}^{p}}\right)_{i, j=1}^{p} \prod_{k=1}^{p-1} \operatorname{det}\left(e^{-* \hat{\pi}_{k+1}\left(x_{j}^{k+1}-x_{i}^{k}\right)}\right)_{i, j=1}^{k+1} e^{-\hat{\pi}_{1} x_{1}^{1}}
$$

Here we used the convention that $x_{k+1}^{k}=0$ for any $1 \leq k \leq p-1$, and the notation

$$
e^{-* \hat{\pi}(x-y)}= \begin{cases}e^{-\hat{\pi}(x-y)}, & x>y \\ 0, & \text { otherwise }\end{cases}
$$

The probability distribution (14) naturally arises here since the distribution of $\max _{j=1, \ldots, p}\left\{x_{j}^{p}\right\}$ is equal to the probability density function of $Y(p, p)$ (see Formula (100).

Let $\mathcal{C}$ (resp. $\mathcal{C}^{\prime}$ ) be a contour encircling the $\left\{\pi_{j}\right\}_{j=1, \ldots, p}\left(\right.$ resp. $\left.\left\{-\hat{\pi}_{j}\right\}_{j=1, \ldots, p}\right)$ such that the two contours do not cross or contain each other. Set

$$
\Psi_{r, s}(u, v)=1_{r<s} 1_{u<v} \frac{1}{2 \pi i} \oint_{\mathcal{C}^{\prime}} e^{w(v-u)} \prod_{k=r+1}^{s} \frac{1}{w+\hat{\pi}_{k}} d w
$$

The main result of this section is the following statement.

Theorem 3. The random point process on $\{1, \ldots, p\} \times \mathbb{R}$ defined by the density (14) is determinantal, and its correlation kernel has the form

$$
\begin{aligned}
& K(r, u ; s, v) \\
& =\frac{1}{(2 \pi i)^{2}} \oint_{\mathcal{C}} d z \oint_{\mathcal{C}^{\prime}} d w \frac{e^{w v-z u}}{w-z} \frac{\prod_{k=1}^{r}\left(z+\hat{\pi}_{k}\right)}{\prod_{l=1}^{s}\left(w+\hat{\pi}_{l}\right)} \prod_{i=1}^{p} \frac{w-\pi_{i}}{z-\pi_{i}}-\Psi_{r, s}(u, v) .
\end{aligned}
$$

Let us briefly discuss the connection with the (discrete) Schur process. A version of the Schur process has a natural interpretation in terms of the last passage percolation model discussed in Section 2. Let again $W=W^{(p)}$ be the $p \times p$ matrix filled with geometrically distributed integers, and let $W^{(k)}$, $k<p$, be the $p \times k$ matrix made of first $k$ columns of $W$. Denote by $\lambda^{(k)}$ the image of $W^{(k)}$ under the Robinson-Schensted-Knuth correspondence. Then 
the joint distribution of $\left(\lambda^{(1)}, \ldots \lambda^{(p)}\right)$ is given by the Schur process: It has the form

$$
\text { const } \cdot s_{\lambda^{(p)}}\left(a_{1}, \ldots, a_{p}\right) s_{\lambda^{(p)} / \lambda^{(p-1)}}\left(b_{p}\right) s_{\lambda^{(p-1)} / \lambda^{(p-2)}}\left(b_{p-1}\right) \cdots s_{\lambda^{(2)} / \lambda^{(1)}}\left(b_{1}\right),
$$

where the notation $s_{\lambda / \mu}$ stands for the skew Schur function. Considering the case where the $a_{i}$ 's and $b_{j}$ 's approach $1\left(a_{i}=1-\pi_{i} / L, b_{i}=1-\right.$ $\hat{\pi}_{j} / L$ and $L \rightarrow \infty$ ), we can then define the continuous limit of the Schur process, which leads to (14). In the context of the percolation model, the probability distribution (14) can be understood as the joint distribution of the random Young diagrams obtained by the RSK algorithm applied to matrices filled with independent but not identically distributed exponential random variables; the expectation of the $(i, j)$ th entry is equal to $\left(\pi_{i}+\hat{\pi}_{j}\right)^{-1}$.

Theorem 3 could be derived from a limiting argument for the correlation kernel of the Schur process, but we prefer to give a self-contained random matrix oriented proof of Theorem 3 below.

The probability distribution (14) can also be viewed in the random matrix theory context of the previous section. For $1 \leq k \leq p$, define $X_{k}$ to be the $p \times k$ matrix whose $k$ columns are the first $k$ columns of $X_{p}$. Then, $M_{k}=X_{k} X_{k}^{*}$ is a $p \times p$ random matrix of rank $k$. We denote by $x_{i}^{k}, 1 \leq i \leq k$ its nonzero eigenvalues. The formula (14) provides a good candidate for the joint distribution density of $\left\{x_{i}^{k}\right\}$ in the sense that its projections to $\left\{x_{i}^{(k)}\right\}$ with fixed $k$ coincide with the densities of eigenvalues of $M_{k}$. Although we were unable to verify that (14) is indeed the joint eigenvalue density for $\left(M_{1}, \ldots, M_{p}\right)$, see the footnote on page 3 for a reference to a partial result.

Proof of Theorem 3. We first consider an (algebraically simpler) auxiliary distribution and then use an appropriate limit transition to compute the correlation functions associated to (14).

Instead of (14) let us consider the probability distribution defined as follows. Let $0<T_{1}<T_{2}<\cdots<T_{p-1}<T_{p}$ be positive numbers, and $T_{1}=\hat{\pi}_{1}$. Define a probability distribution on $\left(\mathbb{R}_{+}^{p}\right)^{p}=\left\{x_{l}^{k}\right\}_{k, l=1 \ldots, p}$ by the density

$$
\frac{1}{Z_{p}^{T}} \operatorname{det}\left(\phi_{0,1}\left(x_{0}^{i}, x_{1}^{j}\right)\right)_{i, j=1}^{p} \prod_{r=1}^{p-1} \operatorname{det}\left(\phi_{r, r+1}\left(x_{r}^{i}, x_{r+1}^{j}\right)\right)_{i, j=1}^{p} \operatorname{det}\left(\phi_{p, p+1}\left(x_{p}^{i}, x_{p+1}^{j}\right)\right)_{i, j=1}^{p}
$$


where $\phi_{0,1}\left(x_{0}^{i}, x\right)=e^{-* T_{i} x}, \phi_{r, r+1}(x, y)=e^{-* \hat{\pi}_{r+1}(y-x)}$ and $\phi_{p, p+1}\left(x, x_{p+1}^{j}\right)=$ $e^{-* \pi_{j} x}$. Set

$$
\begin{aligned}
\Psi_{0, s}^{T}\left(x_{0}^{i}, v\right)= & \int_{\mathbb{R}_{+}^{s-1}} \phi_{0,1}\left(x_{0}^{i}, x_{1}\right) \\
& \left(\prod_{k=1}^{s-2} \phi_{k, k+1}\left(x_{k}, x_{k+1}\right)\right) \phi_{s-1, s}\left(x_{s-1}, v\right) \prod_{i=1}^{s-1} d x_{i}, \\
\Psi_{r, p+1}\left(u, x_{p+1}^{j}\right)= & \int_{\mathbb{R}_{+}^{p-r}}^{p-1} \phi_{r, r+1}\left(u, x_{r+1}\right) \\
& \left(\prod_{k=r+1}^{p-1} \phi_{k, k+1}\left(x_{k}, x_{k+1}\right)\right) \phi_{p, p+1}\left(x_{p}, x_{p+1}^{j}\right) \prod_{i=r+1}^{p} d x_{i}, \\
\Psi_{r, s}(u, v)= & 1_{r<s} \int_{\mathbb{R}_{+}^{s-r}}^{s-2} \phi_{r, r+1}\left(u, x_{r}\right) \\
& \left(\prod_{k=r+1}^{s-1} \phi_{k, k+1}\left(x_{k}, x_{k+1}\right)\right) \phi_{s-1, s}\left(x_{s-1}, v\right) \prod_{i=r} d x_{i} .
\end{aligned}
$$

Lemma 1. For any $1 \leq i, j \leq p$ and $u, v>0$ we have

$$
\begin{aligned}
& \Psi_{0, s}^{T}\left(x_{0}^{i}, v\right)=\frac{1}{2 \pi i} \oint \frac{e^{w v}}{w+T_{i}} \prod_{k=2}^{s} \frac{1}{w+\hat{\pi}_{k}} d w \\
& \Psi_{r, p+1}\left(u, x_{p+1}^{j}\right)=e^{-\pi_{j} u} \prod_{k=r+1}^{p} \frac{1}{\pi_{j}+\hat{\pi}_{k}}, \\
& A_{i j}:=\int_{0}^{+\infty} \Psi_{0, s}^{T}\left(x_{0}^{i}, u\right) \Psi_{r, p+1}\left(u, \pi_{j}\right) d u=\frac{1}{T_{i}+\pi_{j}} \prod_{k=2}^{p} \frac{1}{\pi_{j}+\hat{\pi}_{k}}, \\
& \Psi_{r, s}(u, v)=1_{r<s} 1_{u<v} \frac{1}{2 \pi i} \oint e^{w(v-u)} \prod_{k=r+1}^{s} \frac{1}{w+\hat{\pi}_{k}} d w .
\end{aligned}
$$

The integration contours are positively oriented loops that contain all poles of the integrands.

The proof of Lemma 1 consists of inductions on $s$ for $\Psi_{0, s}^{T}\left(x_{0}^{i}, v\right)$, on $(p-r)$ for $\Psi_{r, p+1}\left(u, x_{p+1}^{j}\right)$, and on $(s-r)$ for $\Psi_{r, s}(u, v)$. The formula for $A_{i j}$ is proved by a straightforward residue computation.

Let us now apply the Eynard-Mehta theorem (see [9], 20, 26], 18, [5]) to compute the correlation functions of (17). For $1 \leq r, s \leq p$, denote

$$
\Psi_{r, s}^{T}(u, v)=1_{r<s} \int_{\mathbb{R}_{+}^{s-r}} \phi_{r, r+1}\left(u, x_{r}\right)
$$




$$
\left(\prod_{k=r+1}^{s-2} \phi_{k, k+1}\left(x_{k}, x_{k+1}\right)\right) \phi_{s-1, s}\left(x_{s-1}, v\right) \prod_{i=r}^{s-1} d x_{i} .
$$

Proposition 2. The random point process on $\{1, \ldots, p\} \times \mathbb{R}_{+}$defined by the measure (17) is determinantal, and its correlation kernel can be written in the form

$$
\begin{aligned}
K_{T}(r, u ; s, v)= & \frac{1}{(2 \pi i)^{2}} \oint_{\mathcal{C}_{1}} d z \oint_{\mathcal{C}_{1}^{\prime}} d w \frac{e^{w v-z u}}{w-z} \\
& \frac{\prod_{k=2}^{r}\left(z+\hat{\pi}_{k}\right)}{\prod_{k=2}^{s}\left(w+\hat{\pi}_{k}\right)} \prod_{i=1}^{p} \frac{\left(w-\pi_{i}\right)\left(z+T_{i}\right)}{\left(z-\pi_{i}\right)\left(w+T_{i}\right)}-\Psi_{r, s}(u, v),
\end{aligned}
$$

where the contour $\mathcal{C}_{1}$ encircles the $\pi_{j}, j=1, \ldots, p$, the contour $\mathcal{C}_{1}^{\prime}$ encircles the $-\hat{\pi}_{j},-T_{j}$, for $j=1, \ldots, p$, and the two contours do not cross or contain each other.

Proof of Proposition 2; $\quad$ The Eynard-Mehta theorem implies that the random point process in question is determinantal, and that its correlation kernel can be written as

$$
K_{T}(r, u ; s, v)=\sum_{i, j=1}^{p} \Psi_{r, p+1}\left(u, x_{p+1}^{i}\right) A_{i j}^{-1} \Psi_{0, s}^{T}\left(x_{0}^{j}, v\right)-\Psi_{r, s}(u, v) .
$$

Using the formula for the determinant of the Cauchy matrix one explicitly computes $A_{i j}^{-1}$, which together with the formula for $\Psi_{0, s}^{T}$ from Lemma 1 yields

$$
\begin{aligned}
& K_{T}(r, u ; s, v)+\Psi_{r, s}(u, v)= \\
& \sum_{i=1}^{p} \Psi_{r, p+1}\left(u, x_{p+1}^{i}\right) \frac{1}{2 \pi i} \oint e^{w v} \frac{\prod_{k=2}^{p}\left(\pi_{i}+\hat{\pi}_{k}\right)}{\prod_{k=2}^{s}\left(w+\hat{\pi}_{k}\right)} \prod_{j=1}^{p} \frac{\pi_{i}+T_{j}}{w+T_{j}} \prod_{k \neq i} \frac{w-\pi_{k}}{\pi_{i}-\pi_{k}} d w
\end{aligned}
$$

where the contour contains all poles of the integrand. A final residue computation yields the integral expression (18).

We can now come back to the computation of correlation functions for the probability distribution (14) and finish the proof of Theorem 3 , The probability distribution (14) can be obtained from (17) by taking the limit $T_{p}>T_{p-1}>\cdots>T_{2} \rightarrow \infty$. The proof of Theorem 3 is now a straightforward corollary of Proposition 2. 


\section{An extension of the Airy point process}

In this section, we first consider the case where $\hat{\pi}_{i}=0$ and $\pi_{i}=1$ for any $i=1, \ldots, p$. We then show that in this case, the suitably rescaled correlation functions converge in the large- $p$-limit to those defined by the extended Airy kernel. Then, to define a new extended Airy-type kernel with parameters, we will allow a certain number of these parameters to depend on $p$ and study the rescaled correlation functions.

\subsection{The simple case $\hat{\pi}_{i}=0$ and $\pi_{i}=1$ for any $i$ : the extended Airy kernel.}

The extended Airy kernel is an extension of the well-known Airy kernel; it occurs for example as the limiting correlation kernel for the process of largest eigenvalues of Dyson's Brownian Motion on Hermitian matrices.

Lemmas 2 and 3 proved in this section are also a part of Proposition 5 of [11].

Let $K_{A i}\left(t_{1}, x ; t_{2}, y\right)$ be the extended Airy kernel defined in (3). Here we show that, when suitably rescaled, the asymptotics of the correlation kernel

$$
\begin{aligned}
K_{p}(r, u ; s, v)= & \frac{p}{(2 \pi i)^{2}} \oint_{\mathcal{C}_{1}} d z \oint_{\mathcal{C}_{1}^{\prime}} d w \frac{e^{p w v-p z u}}{w-z} \\
& \frac{\prod_{k=1}^{r}\left(z+\hat{\pi}_{k}\right)}{\prod_{k=1}^{s}\left(w+\hat{\pi}_{k}\right)} \prod_{i=1}^{p} \frac{w-\pi_{i}}{z-\pi_{i}}-p \Psi_{r, s}(p u, p v),
\end{aligned}
$$

which is a rescaled version of (15), is given by the extended Airy kernel. Due to the choice of the $\pi_{i}$ 's, and $\hat{\pi}_{j}$ 's, one can write that

$$
\begin{aligned}
\tilde{K}_{p}(r, u ; s, v): & =K_{p}(r, u ; s, v)+p \Psi_{r, s}(p u, p v) \\
& =\frac{p}{(2 i \pi)^{2}} \int_{\mathcal{C}_{1}} d z \int_{\mathcal{C}_{1}^{\prime}} d w e^{p\left(F_{v, s}(w)-F_{u, r}(z)\right)} \frac{1}{w-z},
\end{aligned}
$$

where $F_{u, r}(z)=u z+\ln (z-1)-\frac{r}{p} \ln z$.

Let $0<t<1$ be some given real number independent of $p$. Let also $t_{1}, t_{2}$ be given. In the following, we set

$$
\begin{gathered}
r=\left[t p+p^{2 / 3} \frac{2 \sqrt{t}(1+\sqrt{t})^{2}}{\alpha} t_{1}\right]:=s_{1} p, \\
s=\left[t p+p^{2 / 3} \frac{2 \sqrt{t}(1+\sqrt{t})^{2}}{\alpha} t_{2}\right]:=s_{2} p \\
\alpha=\frac{(1+\sqrt{t})^{4 / 3}}{t^{1 / 6}}, \quad u=\left(1+\sqrt{s_{1}}\right)^{2}+\frac{\alpha x}{p^{2 / 3}}, \quad v=\left(1+\sqrt{s_{2}}\right)^{2}+\frac{\alpha y}{p^{2 / 3}} .
\end{gathered}
$$


Here $[x]$ stands for the integral part of $x \in \mathbb{R}$.

We first consider the case where $s \leq r$. Set $\Gamma:=\left\{t e^{ \pm 2 i \pi / 3}, t \in \mathbb{R}_{+}\right\}$to be a contour oriented from bottom to top and $\gamma:=\left\{t e^{ \pm i \pi / 3}, t \in \mathbb{R}_{+}\right\}$to be oriented from top to bottom.

Lemma 2. With the above rescaling, for $s \leq r$ and $z_{0}:=\frac{\sqrt{t}}{1+\sqrt{t}}$ we have

$$
\begin{aligned}
& \lim _{p \rightarrow \infty} \frac{\alpha}{p^{2 / 3}} e^{p\left(F_{u, r}\left(z_{0}\right)-F_{v, s}\left(z_{0}\right)\right)} \tilde{K}_{p}(r, u ; s, v) \\
& =\frac{e^{y t_{2}-x t_{1}+\frac{1}{3}\left(t_{1}^{3}-t_{2}^{3}\right)}}{(2 \pi i)^{2}} \int_{\gamma} d s^{\prime} \int_{\Gamma} d t^{\prime} \frac{e^{y t^{\prime}-\frac{t^{\prime 3}}{3}-x s^{\prime}+\frac{s^{\prime 3}}{3}}}{t^{\prime}-s^{\prime}+t_{2}-t_{1}} \\
& =e^{y t_{2}-x t_{1}+\frac{1}{3}\left(t_{1}^{3}-t_{2}^{3}\right)} \int_{0}^{\infty} e^{-\lambda\left(t_{1}-t_{2}\right)} A i(y+\lambda) A i(x+\lambda) d \lambda .
\end{aligned}
$$

Proof of Lemma 2. It is convenient to define $u_{o}=\left(1+\sqrt{s_{1}}\right)^{2}$ and $v_{o}=$ $\left(1+\sqrt{s_{2}}\right)^{2}$. The reason for the above rescaling (20) $-(22)$ is that

$$
\begin{aligned}
F_{u, r}(z) & =F_{u_{o}, r}(z)(1+o(1)) \\
& =\left(u_{o} z+\ln (z-1)-s_{1} \ln z\right)(1+o(1)):=f_{s_{1}}(z)(1+o(1)),
\end{aligned}
$$

where the function $f_{s_{1}}(z)$ has a degenerate critical point at

$$
z_{c}:=\frac{\sqrt{s_{1}}}{1+\sqrt{s}_{1}} \sim z_{0}+O\left(p^{-1 / 3}\right) .
$$

In particular, one has that:

$$
f_{s_{1}}^{\prime}\left(z_{c}\right)=f_{s_{1}}^{\prime \prime}\left(z_{c}\right)=0, \text { and } f_{s_{1}}^{\prime \prime \prime}\left(z_{c}\right)=\frac{-2\left(1+\sqrt{s_{1}}\right)^{4}}{\sqrt{s_{1}}} .
$$

To obtain the leading term in the asymptotic expansion of $K_{p}$, we define the following contours. Using the notation $w_{c}=\frac{\sqrt{s_{2}}}{1+\sqrt{s_{2}}}$, set

$$
\mathcal{C}_{1,1}=\left\{z_{c}+s^{\prime} e^{ \pm i \pi / 3}, 0 \leq s^{\prime}<\delta_{o}\right\}, \mathcal{C}_{1,1}^{\prime}=\left\{w_{c}+t^{\prime} e^{ \pm i 2 \pi / 3}, 0 \leq t^{\prime}<\delta_{1}\right\},
$$

where $\delta_{o}>0$ and $\delta_{1}>0$ are constants that will be determined in the sequel. These contours are completed as follows. Set $\theta_{o}=\arg \left(z_{c}+\delta_{o} e^{i \pi / 3}\right)$ (resp. $\left.\theta_{1}=\arg \left(w_{c}+\delta_{1} e^{2 i \pi / 3}\right)\right)$, where $\arg$ denotes the argument of a complex number. Set

$$
\left.\mathcal{C}_{1,2}^{\prime}=\left\{\left|w_{c}+\delta_{1} e^{2 i \pi / 3}\right| e^{i \theta}, \theta_{1} \leq \theta \leq 2 \pi-\theta_{1}\right]\right\}
$$




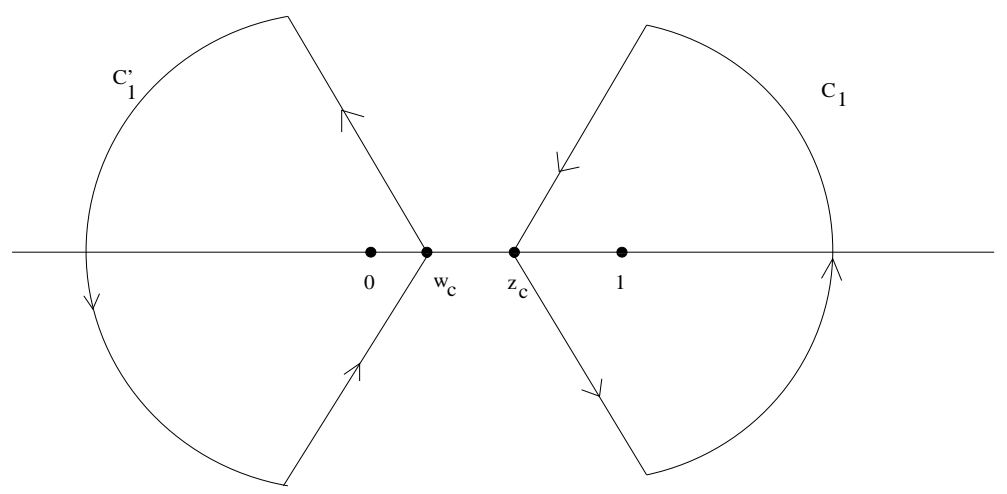

Figure 2: The two contours $\mathcal{C}_{1}$ and $\mathcal{C}_{1}^{\prime}$

$$
\mathcal{C}_{1,2}=\left\{1+\left|z_{c}+\delta_{o} e^{i \pi / 3}-1\right| e^{i \theta},|\theta| \leq \theta_{o}\right\} .
$$

The constant $\delta_{o}$ is also large enough so that the $z$-contour encircles all the $\pi_{i}$ 's (even in the case where some of them differ from 1 ). It can then be checked that

$$
\frac{\partial}{\partial s} \Re\left(f_{s_{2}}\left(w_{c}+\frac{s e^{2 i \pi / 3}}{1+\sqrt{s}_{2}}\right)\right)=-\frac{s^{2}\left(1+\sqrt{s_{2}}\right)\left(s^{2}-s\left(1-\sqrt{s_{2}}\right)+2 \sqrt{s_{2}}\right)}{2\left(s^{2}+s+1\right)\left(s^{2}-s \sqrt{s_{2}}+s_{2}\right)}<0
$$

for any $s>0$ provided $\sqrt{s_{2}}>5-\sqrt{96} / 2$. If $\sqrt{s_{2}}<5-\sqrt{96} / 2$, we set $\delta_{1}$ to be the smallest positive root of the polynomial $X^{2}-X\left(1-\sqrt{s_{2}}\right)+2 \sqrt{s_{2}}$. Otherwise $\delta_{1}$ is arbitrarily large. Similarly

$$
\frac{\partial}{\partial s} \Re\left(f_{s_{1}}\left(z_{c}+\frac{s e^{i \pi / 3}}{1+\sqrt{s}_{1}}\right)\right)=\frac{s^{2}\left(1+\sqrt{s_{1}}\right)\left(s^{2}+s\left(1-\sqrt{s_{1}}\right)+2 \sqrt{s_{1}}\right)}{2\left(s^{2}-s+1\right)\left(s^{2}+s \sqrt{s_{1}}+s_{1}\right)}>0,
$$

for any $s>0$. We also have that if $|\delta|$ is bounded

$$
\left|p f_{s_{2}}\left(w_{c}+\frac{\delta}{p^{1 / 3}}\right)-p f_{s_{2}}\left(w_{c}\right)-f_{s_{2}}^{(3)}\left(w_{c}\right) \frac{\delta^{3}}{3 !}\right| \leq \frac{\sup _{\Omega}\left|f_{s_{1}}^{(4)}(w)\right|}{p^{1 / 3}} \leq C \frac{\delta^{4}}{p^{1 / 3}} .
$$

Here $w=w_{c}+\frac{\delta}{p^{1 / 3}}$ lies in a compact subset $\Omega$ of $\mathbb{C} \backslash\{0,1\}$.

To complete the proof one needs to verify that on remaining parts of the contours the integrand becomes exponentially small as $p$ gets large. If we set $w:=\left|w_{c}+\delta_{1} e^{2 i \pi / 3}\right| e^{i \theta}$, and using the fact that $v_{o}=\left|w_{c}-1\right|^{-2}$, one can check that

$$
\Re\left(\frac{\partial}{\partial \theta} f_{s_{2}}(w)\right)=-\Im(w)\left(v_{o}-\frac{1}{|w-1|^{2}}\right)<0,
$$


if $\theta \in\left[\theta_{1}, \pi\right]$. It is an easy computation to check the remaining parts of the contours, and we omit it.

If we assume that $t_{1}, t_{2}, x, y$ lie in a compact set of $\mathbb{R}$, then the above estimates imply that

- It is enough to integrate over a neighborhood of radius $p^{-1 / 3} p^{1 / 12-\epsilon}$ of the critical points.

- Inside such a neighborhood the Taylor expansion holds.

Writing out this expansion explicitly yields Lemma 2,

In the case $s>r$ we cannot make the integration contours go through their corresponding critical points so that they do not intersect. We then modify the contours in a neighborhood of width $p^{-1 / 3}$ of $z_{c}$ and $w_{c}$ so that the $w$-contour remains on the left of the $z$-contour. This does not modify the saddle point argument. We just need to consider the function $\Psi_{r, s}$ separately, which is done below.

Due to the rescaling of the correlation kernel, in the case where $r<s$, we need to consider the asymptotics of

$$
\frac{\alpha}{p^{2 / 3}} e^{p\left(F_{u, r}\left(z_{0}\right)-F_{v, s}\left(z_{0}\right)\right)} \frac{p}{2 \pi i} \oint_{\gamma_{o}} e^{p w(v-u)} \frac{d w}{w^{s-r}}
$$

where $\gamma_{o}$ is a contour encircling the pole $w=0$.

Lemma 3. For $s>r$, using the scaling (20)-(22) one has

$$
\begin{aligned}
& \lim _{p \rightarrow \infty} \frac{\alpha}{p^{2 / 3}} e^{p F_{u, r}\left(z_{0}\right)-p F_{v, s}\left(z_{0}\right)} K_{p}(r, u ; s, v) \\
& =-e^{y t_{2}-x t_{1}+\frac{\left(t_{1}^{3}-t_{2}^{3}\right)}{3}} \int_{-\infty}^{0} e^{-\lambda\left(t_{1}-t_{2}\right)} A i(y+\lambda) A i(x+\lambda) d \lambda .
\end{aligned}
$$

Proof of Lemma 3; $\quad$ Setting $\beta=2 \sqrt{t}(1+\sqrt{t})^{2} / \alpha$, one has

$$
\begin{aligned}
& \frac{\alpha}{p^{2 / 3}} e^{\left\{p F_{u, r}\left(z_{0}\right)-p F_{v, s}\left(z_{0}\right)\right\}} \frac{p}{2 \pi i} \oint_{\gamma_{o}} e^{p w(v-u)} \frac{d w}{w^{s-r}} \\
& =\frac{\alpha}{p^{2 / 3}} e^{\left\{p F_{u, r}\left(z_{0}\right)-p F_{v, s}\left(z_{0}\right)\right\}} \frac{p}{2 \pi i} \oint_{\gamma_{o}} d w \frac{\exp \left\{p^{2 / 3} \frac{(1+\sqrt{t}) \beta\left(t_{2}-t_{1}\right)}{\sqrt{t}} w\right\}}{w^{\beta p^{2 / 3}\left(t_{2}-t_{1}\right)+O(1)}} \\
& \times \exp \left\{p^{1 / 3}\left(-\frac{\beta^{2}}{4 t^{3 / 2}}\left(t_{2}^{2}-t_{1}^{2}\right) w+\alpha(y-x) w+o(1)\right)\right\} .
\end{aligned}
$$


Consider

$$
F(w)=\beta\left(t_{2}-t_{1}\right) \frac{1+\sqrt{t}}{\sqrt{t}} w-\beta\left(t_{2}-t_{1}\right) \ln w
$$

It is not hard to see that the critical point of this function is $w_{0}=z_{0}=\frac{\sqrt{t}}{1+\sqrt{t}}$ and $F^{\prime \prime}\left(z_{0}\right)=\beta\left(t_{2}-t_{1}\right) z_{0}^{-2}$. A contour which satisfies the saddle point analysis requirement can be chosen as follows: $\gamma_{o}=\gamma_{1} \cup \overline{\gamma_{1}}$ where $\gamma_{1}=$ $\left\{z_{0}+i t,|t| \leq z_{0}\right\} \cup\left\{z_{0} e^{i(\pi / 4+\theta)}, 0<\theta<3 \pi / 4\right\}$.

We obtain

$$
\begin{aligned}
& \lim _{p \rightarrow \infty} \frac{\alpha}{p^{2 / 3}} \exp \left\{p F_{u, r}\left(z_{0}\right)-p F_{v, s}\left(z_{0}\right)\right\} \frac{p}{2 \pi i} \oint_{\gamma_{o}} e^{p w(v-u)} \frac{d w}{w^{s-r}} \\
= & \lim _{p \rightarrow \infty} \frac{\alpha}{2 \pi i} \frac{1}{\sqrt{F^{\prime \prime}\left(z_{0}\right)}} \int_{i \mathbb{R}} \exp \left\{\frac{s^{\prime}\left(\alpha(y-x)-\frac{\beta^{2}\left(t_{2}^{2}-t_{1}^{2}\right)}{4 t^{3 / 2}}\right)}{\sqrt{F^{\prime \prime}\left(z_{0}\right)}}+\frac{s^{\prime 2}}{2}\right\} d s^{\prime} \\
= & \frac{1}{2 \pi i \sqrt{2\left(t_{2}-t_{1}\right)}} \int_{i \mathbb{R}} \exp \left\{\frac{s^{\prime 2}}{2}+\frac{(y-x) s^{\prime}-\left(t_{2}^{2}-t_{1}^{2}\right) s^{\prime}}{\sqrt{2\left(t_{2}-t_{1}\right)}}\right\} d s^{\prime} \\
= & \frac{1}{\sqrt{4 \pi\left(t_{2}-t_{1}\right)}} \exp \left\{-\frac{\left(y-x+t_{1}^{2}-t_{2}^{2}\right)^{2}}{t_{2}-t_{1}}\right\} .
\end{aligned}
$$

Proposition 2.3 of [18] completes the proof.

\subsection{Extended Airy kernel with two sets of parameters}

We now consider the case where some of the $\pi_{i}$ 's (resp. $\hat{\pi}_{j}$ 's) differ from 1 (resp. 0). This allows us to obtain a new extended Airy type kernel with two sets of parameters and prove Theorem 2 .

We assume that (7) holds true and that all the $x_{i}$ 's and $y_{i}$ 's lie in a fixed compact set of $\mathbb{R}$. We also assume that $x_{i}-y_{j}>0$ for any $i, j$, so that the joint distribution (14) is well defined.

Theorem 4. With the above rescaling, one has

$$
\lim _{p \rightarrow \infty} \frac{\alpha}{p^{2 / 3}} e^{p F_{u, r}\left(z_{0}\right)-p F_{v, s}\left(z_{0}\right)} K_{p}(r, u ; s, v)=\text { (4) }
$$

where the integration contours $\gamma$ and $\Gamma$ are chosen as in Figure 1 .

Remark 3. Theorem 4 readily implies Theorem 2 
Proof of Theorem 4; The proof relies on the same saddle point analysis of the correlation kernel as in the previous section. In the expression (19) we replace

$$
\frac{1}{w-z} \frac{\prod_{k=1}^{r}\left(z+\hat{\pi}_{k}\right)}{\prod_{k=1}^{s}\left(w+\hat{\pi}_{k}\right)} \prod_{i=1}^{p} \frac{w-\pi_{i}}{z-\pi_{i}}
$$

by

$$
\frac{\prod_{k=J_{2}+1}^{r}\left(z+\hat{\pi}_{k}\right)}{\prod_{k=J_{2}+1}^{s}\left(w+\hat{\pi}_{k}\right)} \prod_{i=J_{1}+1}^{p} \frac{w-\pi_{i}}{z-\pi_{i}}\left(\frac{1}{w-z}+\frac{\left(\prod_{k=1}^{J_{2}} \frac{z+\hat{\pi}_{k}}{w+\hat{\pi}_{k}} \prod_{i=1}^{J_{1}} \frac{w-\pi_{i}}{z-\pi_{i}}-1\right)}{w-z}\right)
$$

and observe that the second summand has no singularity at $z=w$. This allows us to use the same contour deformation as in the previous section, which directly leads to the result.

\section{References}

[1] Baik, J.; Deift, P. and Johansson, K. (1999). On the distribution of the length of the longest increasing subsequence of random permutations. J. Amer. Math. Soc. 12, no. 4, 1119-1178.

[2] Baik, J. Ben Arous, G. and Peche, S. (2006) Phase transition of the largest eigenvalue for nonnull complex sample covariance matrices. Ann. of Prob. 33, 1643-1697.

[3] Borodin, A. (2007). Periodic Schur process and cylindric partitions. Duke Math. Jour. 140, no 3, 391-468.

[4] Borodin, A. and Olshanski, G. (2006). Stochastic dynamics related to Plancherel measure on partitions. In: Representation theory, dynamical systems, and asymptotic combinatorics, 9-21, Amer. Math. Soc. Transl. Ser. 2, 217, Amer. Math. Soc., Providence, RI.

[5] Borodin, A. and Rains, E. M. (2006). Eynard-Mehta theorem, Schur process and their Pfaffian analogs. J. Stat. Phys. 121, no. 3-4, 291-317.

[6] Bowick, M. and Brézin, E. (1991). Universal scaling of the tail of the density of eigenvalues in random matrix models. Phys. Lett. B 268, no. 1, 21-28. 
[7] Desrosiers, P. and Forrester, P. J. (2006). Asymptotic correlations for Gaussian and Wishart matrices with external source, Intern. Math. Res. Notices, 43 pages.

[8] EdreI, A.(1953) On the generating function of a doublyinfinite, totally positive sequence. Trans. Amer. Math. Soc. 74, no. 3, 367-383.

[9] Eynard, B. and Mehta, M. L. (1998) Matrices coupled in a chain. I. Eigenvalue correlations. J. Phys. A: Math. Gen. 31, 44494456.

[10] Forrester, P. J. (1993). The spectrum edge of random matrix ensembles. Nuclear Phys. B 402, no. 3, 709-728.

[11] Forrester, P.J. and Nagao, T. (2008) Determinantal correlations for classical projection processes. Preprint, available at arXiv:0801.0100.

[12] Forrester, P.J. and Rains, E.M. (2005) Interpretations of some parameter dependent generalizations of classical matrix ensembles Prob. Theory and Relat. Fields 131, 1-61.

[13] Glynn, P and Whitt, W. (1991). Departures from many queues in series. Ann. Appl. Probab. 1, no. 4, 546-572.

[14] Imamura, T. and Sasamoto, T. (2007). Dynamics of a tagged particle in the asymmetric exclusion process with the step initial condition. Preprint, available at arXiv:math-ph/0702009.

[15] Johansson, K. (2005). Random matrices and determinantal processes. Lectures given at the summer school on Mathematical statistical mechanics in July 05 at Ecole de Physique, Les Houches. Available at: arXiv:math-ph/0510038.

[16] Johansson, K. (2001). Random growth and random matrices. In:European Congress of Mathematics, Vol. I (Barcelona, 2000), 445456, Progr. Math., 201, Birkhuser, Basel.

[17] Johansson, K. (2000). Shape fluctuations and random matrices. Comm. Math. Phys. 209, 437-476.

[18] Johansson, K. (2003). Discrete polynuclear growth and determinantal processes. Comm. Math. Phys. 242, no. 1-2, 277-329. 
[19] MaCÊDo, A. M.(1994) Universal parametric correlations at the Soft Edge of the spectrum of random matrix ensembles. Eurohys. Lett. 26, no $9,641-646$.

[20] Nagao, T. and Forrester, P. J. (1998). Multilevel Dynamical Correlation Function for Dyson's Brownian Motion Model of Random Matrices. Phys Lett. A 247, 42-46.

[21] Okounkov, A. (2001). Infinite wedge and random partitions. Selecta Math. (N.S.) 7, no. 1, 57-81.

[22] Okounkov, A. and Olshanski, G. (1998) Asymptotics of Jack polynomials as the number of variables goes to infinity. Internat. Math. Res. Notices, no. 13, 641-682.

[23] Okounkov, A. and Reshetikhin, N. (2003) Correlation function of Schur process with application to local geometry of a random 3dimensional Young diagram. J. Amer. Math. Soc. 16, no. 3, 581-603.

[24] Prähofer, M. and Spohn, H. (2002). Scale invariance of the PNG droplet and the Airy process. J. Statist. Phys. 108, no. 5-6, 1071-1106.

[25] Tracy, C. A. and Widom, H. Level-spacing distributions and the Airy kernel. Phys. Lett. B 305, no. 1-2, 115-118.

[26] Tracy, C. A. and Widom, H. (2004). Differential equations for Dyson processes Comm. Math. Phys. 252, no. 1-3, 7-41. 